N. E. Piskunov, W. W. Weiss, D. F. Gray, eds.

\title{
Stratified NLTE Model Atmospheres for Hot Stars
}

\author{
Stefan Dreizler and Sonja L. Schuh \\ Institut für Astronomie und Astrophysik, Universität Tübingen, Sand 1, \\ D-72076 Tübingen
}

\begin{abstract}
In most stellar atmosphere models, a homogeneous chemical composition is assumed, which is a good approximation for many stars. With the absence of convection and mass-loss, however, gravitational settling can produce a chemical stratification gradient. Typical examples are white dwarfs. In hot stars this diffusion process is modified by the radiative acceleration, resulting in levitation of ions with large radiative cross sections. The status of our program for self-consistent stratified non-LTE model atmospheres is presented.
\end{abstract}

\section{Introduction}

Element segregation can be observed in different types of stars, e.g. main sequence A stars, subdwarf B stars, or white dwarfs (Michaud 1970, Greenstein et al. 1967, Michaud et al. 1983, Schatzman 1949, 1953). The mono-elemental composition in most white dwarfs is a direct indication for such diffusion processes. The pure hydrogen atmosphere of DA white dwarfs is interpreted as a product of gravitational settling of all heavier elements leaving a very purified atmosphere. In this paper we will concentrate on the white dwarfs because the effects can be most clearly observed in these stars. The hope is that this comparable simplicity will also enable a realistic modeling of the processes.

The high surface gravity $(\log g \approx 8)$ of white dwarfs in combination with very calm atmospheres during long periods of the white dwarf evolution determines the chemical composition of these stars. The lightest element, depending on the evolutionary history either hydrogen or helium, diffuses on top of the atmosphere and dominates the optical spectrum. However, this simple picture is altered if competing mechanisms are present. In the case of white dwarfs hotter than $20000 \mathrm{~K}$ the most important process is selective radiative pressure (radiative levitation). Theoretical calculations for white dwarfs by Vauclair et al. (1979) and later by Morvan et al. (1986), Vauclair (1987, 1989), and Chayer et al. $(1989,1991,1994,1995 \mathrm{a}, 1995 \mathrm{~b})$ have shown that radiative acceleration can be strong enough at some effective temperatures and gravities to provide traces of photospheric elements such as carbon, nitrogen, oxygen, silicon, iron, and nickel. Unglaub \& Bues $(1989,2000,2001)$ investigated the influence of a weak stellar wind on the element stratification of hot white dwarfs $\left(T_{\text {eff }} \gtrsim 65000 \mathrm{~K}\right)$ and subdwarf B stars while self-consistent calculations for Ap stars were presented by Hui-Bon-Hoa et al. (2002). 
These theoretical predictions of abundances for trace elements in white dwarfs can be confronted by numerous observations in the ultraviolet, extremeultraviolet, and X-ray regions of the electromagnetic spectrum. A flux deficit caused by the additional opacity in the EUV as compared to pure hydrogen atmospheres, hinted at already by earlier data (Mewe et al. 1975, Hearn et al. 1976, Lampton et al. 1976, Margon et al. 1976, Shipman 1976), became more and more evident as the quality of observations improved with the HEAO (Einstein), EXOSAT and ROSAT satellites (Kahn et al. 1984, Petre et al. 86, Jordan et al. 1987, Paerels \& Heise 1989, Barstow et al. 1993b, Jordan et al. 1994, Wolff et al. 1996). While one EXOSAT (Vennes et al. 1989) and various ROSAT observations revealed the opacity to be mainly due to absorbers other than helium, observations with EUVE made possible a more detailed investigation of the nature of absorbers. Recent analyses of hot white dwarf EUVE spectra have been presented by Barstow et al. (1997) and Wolff et al. (1998). Using the latest non-LTE and LTE atmosphere models, both groups derived a metal mix scaling factor for each object in their respective WD sample. Barstow et al. (1997) use this single scaling factor per object to adjust metal abundances predicted for its combination of effective temperature and surface gravity by Chayer et al. (1995a,b), whereas Wolff et al. (1998) use relative metal abundances which they have derived for the standard star G 191-B2B as a typical metal mix and give the appropriate scaling factor that they call metallicity for each of their sample objects. There are many UV observations starting with IUE and later continued with HST and FUSE (a complete compilation would be beyond the scope of this paper, we can therefore name only a few, e.g. Bruhweiler \& Kondo 1981, Vennes et al. 1992, Sion et al. 1992, Holberg et al. 1994, Werner \& Dreizler 1994, Vidal-Madjar et al. 1994, Lanz et al. 1996, Vennes et al. 1996, Wolff et al. 1998, Holberg et al. 1999, Chayer et al. 2000, Vennes \& Lanz 2001, Barstow et al. 2001).

This very rich observing material of very high quality was in contrast to the modeling ability for quite a while. The data were analysed mainly with chemical homogeneous model atmosphere because no stratified models were available. These results were later compared to predicted abundances derived from the equilibrium between radiative levitation and gravitative settling (e.g. Chayer et al. 1995a,b). The major drawback of these calculations are the assumption of a fixed flux distribution obtained from homogeneous models for the calculation of the radiative forces. A re-coupling of the chemical stratification on the atmospheric structure was not taken into account. It is therefore not surprising that these results could only provide a rough outline of the chemical evolution of white dwarfs. The shortcomings of the models can now be overcome by calculating the atmospheric structure, the radiation field, and the element distribution self-consistently (Dreizler 1999). We have successfully applied these models to a sample of EUVE spectra of white dwarfs (Dreizler \& Wolff, 1999, Schuh et al. 2002). In the following we will summarize the numerical method and shortly describe our results. 


\section{Outline of the model calculations}

The modeling of stratified NLTE model atmospheres splits in two major parts: On the one hand the self-consistent solution of the radiation transport together with the rate equations provides the radiation field and the the atomic level populations (see Werner \& Dreizler for details and Dreizler 2002 for the newest developments). On the other hand the chemical stratification is determined from the equilibrium between radiative levitation and gravitational settling (see also Dreizler 1999, Hui-Bon-Hoa et al. 2002).

The equilibrium requires the absence of all other competing processes which is very well fulfilled for at least the majority of white dwarfs under investigation:

Rotation produces meridional currents which would counteract the stratification. Rotation velocities are however very low in white dwarfs. Spectroscopically only upper limits of about $v \sin i=10 \mathrm{~km} / \mathrm{s}$ (Heber et al. 1997, Koester et al. 1998) can be derived while asteroseismology provides direct measurements for rotation periods in the order of days, i.e. $v \sin i \sim 1 \mathrm{~km} / \mathrm{s}$ (Kawaler \& Bradley 1994, Bradley \& Winget 1994)

Mass loss drops below a critical limit of $10^{-13} \mathrm{M}_{\odot} / \mathrm{yr}$ (Unglaub \& Bues 1998) for white dwarfs with effective temperatures below about $65000 \mathrm{~K}$ and is therefore unimportant at least for most DA white dwarfs.

Convection is negligible for all hot white dwarfs in the range where radiative acceleration is still sufficient enough to support heavy elements.

The short diffusion time scale in combination with the absence of convection and mass loss allows the determination of abundances at each depth point from the balance of gravitational, radiative and electrical forces. For a trace element $(i)$ in a plasma mainly composed of element (1) this can be written as:

$$
m_{1} F_{1}-m_{i} F_{i}=\left(A_{i}-A_{1}\right) m_{\mathrm{p}} g-\left(Z_{i}-Z_{1}\right) e E-A_{i} m_{\mathrm{p}} g_{\mathrm{rad}, i}=0,
$$

i.e. the fources $m_{1} F_{1}, m_{i} F_{i}$ are balanced and there is no net velocity of element $i$ with respect to element $1 . A_{i}$ are the atomic weights, $Z_{i}$ the ionic electrical charges, $e$ is the charge of an electron, $m_{p}$ is the proton mass, and $g_{\mathrm{rad}, i}$ is the radiative acceleration acting on element $(i)$. Neglecting the radiative forces on element (1) assumes that the main constituent of the plasma is homogeneously distributed over the atmosphere (however, still hydrostatically stratified). The radiative acceleration is given by

$$
g_{\mathrm{rad}, i}=\frac{1}{\rho_{i}} \frac{4 \pi}{c} \int_{0}^{\infty} \kappa_{\nu, i} H_{\nu} \mathrm{d} \nu
$$

where $\rho_{i}$ is the mass fraction of the element (i), $\kappa_{\nu, i}$ is the frequency dependent mass absorption coefficient which includes all contributions of this element at the frequency $\nu$, and $H_{\nu}$ is the Eddington flux. The electric field $E$ can be obtained from charge conservation:

$$
\sum P_{j} Z_{j}=P_{\mathrm{e}}
$$




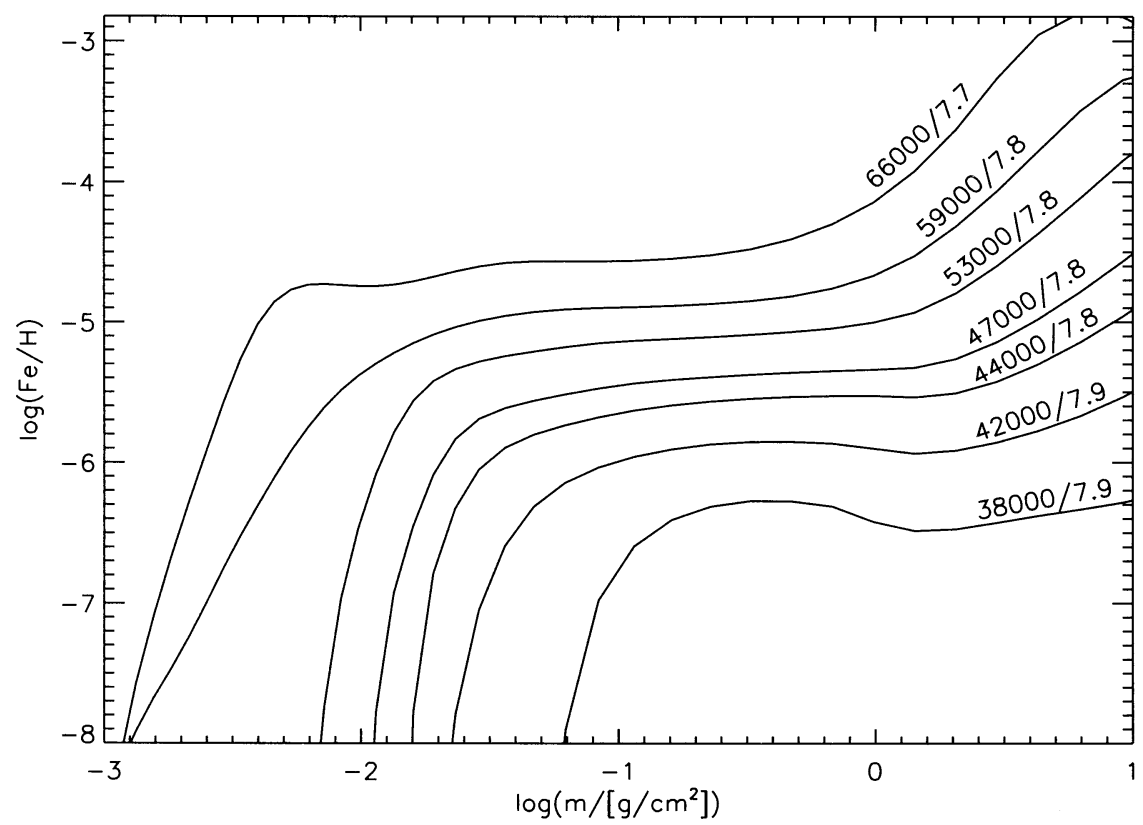

Figure 1. Chemical gradient of iron in models for DA white dwarfs along the cooling track of $0.6 \mathrm{M}_{\odot}$. The models are labeled according to their effective temperature in Kelvin and the logarithm of the surface gravity in cgs units. The atmospheric depth is given in column mass.

where $P_{j}$ and $P_{\mathrm{e}}$ denote the partial pressure of the elements $(j)$ and of the electrons, respectively. Differentiating Eq. (3) with respect to $r$ and inserting the partial pressures $P_{i}$ as given by

$$
\frac{1}{P_{i}} \frac{\mathrm{d} P_{i}}{\mathrm{~d} r}=\frac{A_{i} m_{\mathrm{p}} g}{k T}+\frac{Z_{i} e E}{k T}
$$

results after some approximations in

$$
e E=\frac{A_{1} m_{\mathrm{p}} g}{Z_{1}+1} .
$$

With this expression Eq. (1) reads

$$
\begin{aligned}
m_{1} F_{1}-m_{i} F_{i} & =m_{\mathrm{p}} g\left(A_{i}-A_{1}-\frac{Z_{i} A_{1}}{Z_{1}+1}+\frac{Z_{1} A_{1}}{Z_{1}+1}\right)-A_{i} m_{p} g_{\mathrm{rad}} \\
& =A_{i} m_{\mathrm{p}}\left(\left(1-\frac{A_{1}\left(Z_{i}+1\right)}{A_{i}\left(Z_{1}+1\right)}\right) g-g_{\mathrm{rad}, \mathrm{i}}\right) \\
& =0 .
\end{aligned}
$$

With

$$
g_{\mathrm{eff}, \mathrm{i}}:=\left(1-\frac{A_{1}\left(Z_{i}+1\right)}{A_{i}\left(Z_{1}+1\right)}\right) g
$$




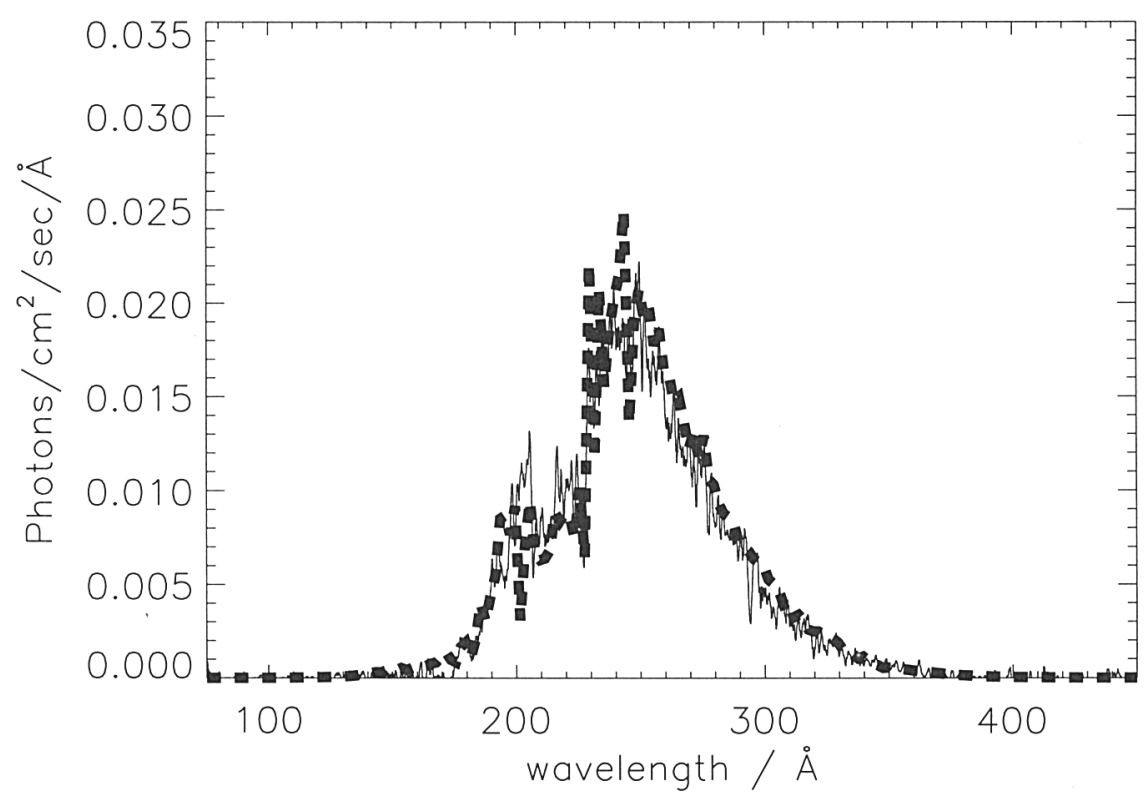

Figure 2. The EUVE spectrum of MCT 2331-4731 (solid) compared with a stratified NLTE model atmosphere (dashed) of $T_{\text {eff }}=56000 \mathrm{~K}$, $\log g=7.6$.

Eq. (4) yields:

$$
g_{\mathrm{rad}, \mathrm{i}}=g_{\mathrm{eff}, \mathrm{i}}
$$

which is exactly what Chayer et al. (1995a) derived. Eq. (5) defines the mass fraction of element $(i)$ at each depth point through the dependence of the radiative acceleration on $\rho_{i}$ (Eq. 2).

In Fig. 1 the development of the chemical gradient of iron in DAs along a cooling track for a $0.6 \mathrm{M}_{\odot}$ white dwarf is demonstrating the interplay between gravitational settling and radiative levitation. The decrease of the effective temperature reduces the radiative acceleration and iron is more and more depleted as the star cools down. It should be noted that the equilibrium formulation requires two input parameters only: The effective temperature and surface gravity. The chemical composition is then determined by the equilibrium.

In contrast to the necessity in homogeneous model atmospheres, the opacity has to be determined accounting for Stark broadening of every line. Additionally, the model atoms have to be very detailed in order to provide a realistic amount of radiative acceleration. In the current stage, we ignore possible effects from redistribution of the transferred momentum over the ionization stages. We also assume that a bound-free transition transfers the momentum completely to the ion ignoring a momentum transfer to the electron. 


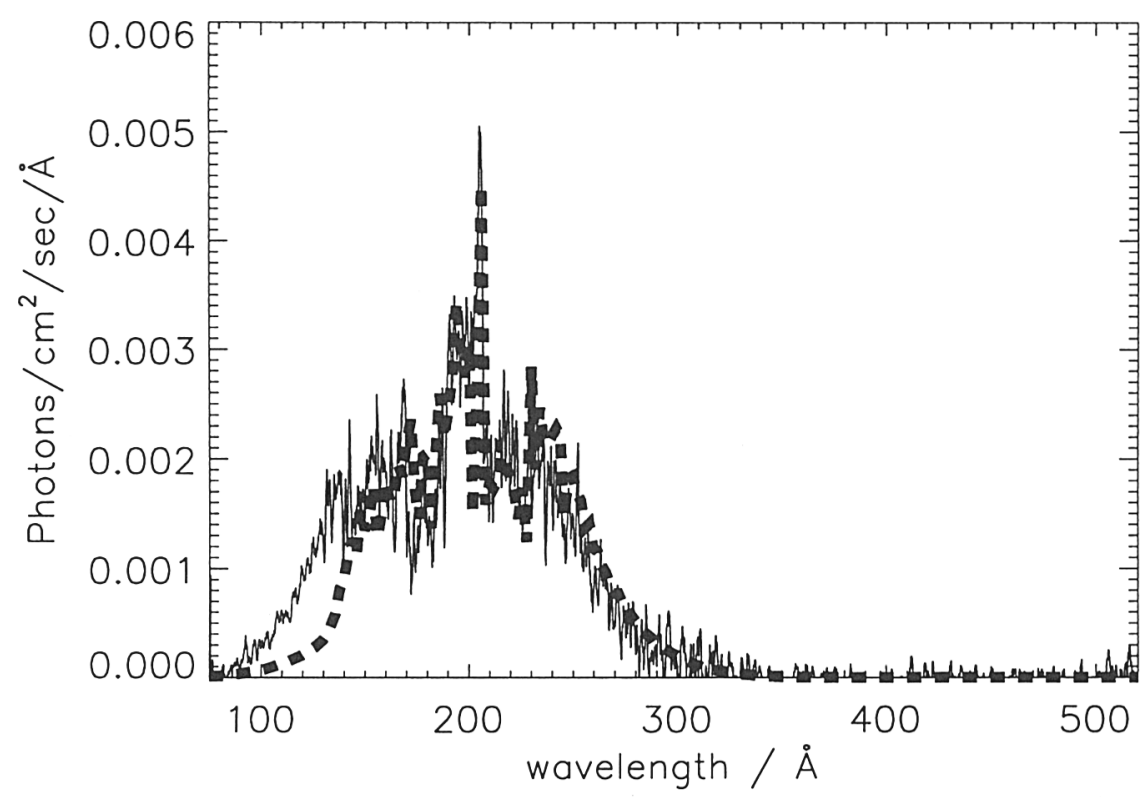

Figure 3. The EUVE spectrum of PG 1123+189 (solid) compared with a stratified NLTE model atmosphere (dashed) of $T_{\text {eff }}=54000 \mathrm{~K}$, $\log g=7.9$.

Since the equilibrium condition (5) is coupled via the opacity and the Eddington flux to the structure of the model atmosphere, it must be solved selfconsistently with our usual set of equations necessary for the construction of non-LTE model atmospheres. We perform this in the most simple way by an iterative scheme. Starting with a homogeneous atmosphere we take its radiation field, density and temperature stratification as well as the occupation numbers of all atomic energy levels and calculate the radiative acceleration of all elements at all depth points. We then solve Eq. (5) to obtain a chemical stratification for all trace elements. Keeping this fixed, we re-determine the structure and the radiation field for the next iteration step. Alternatively the equilibrium condition could be included as an additional constraint equation in the construction of the model atmosphere which in principle would result in a faster convergence. However, starting from the chemically homogeneous models would then be nearly impossible, due to large initial changes in the abundances and therefore in the atmospheric structure. 


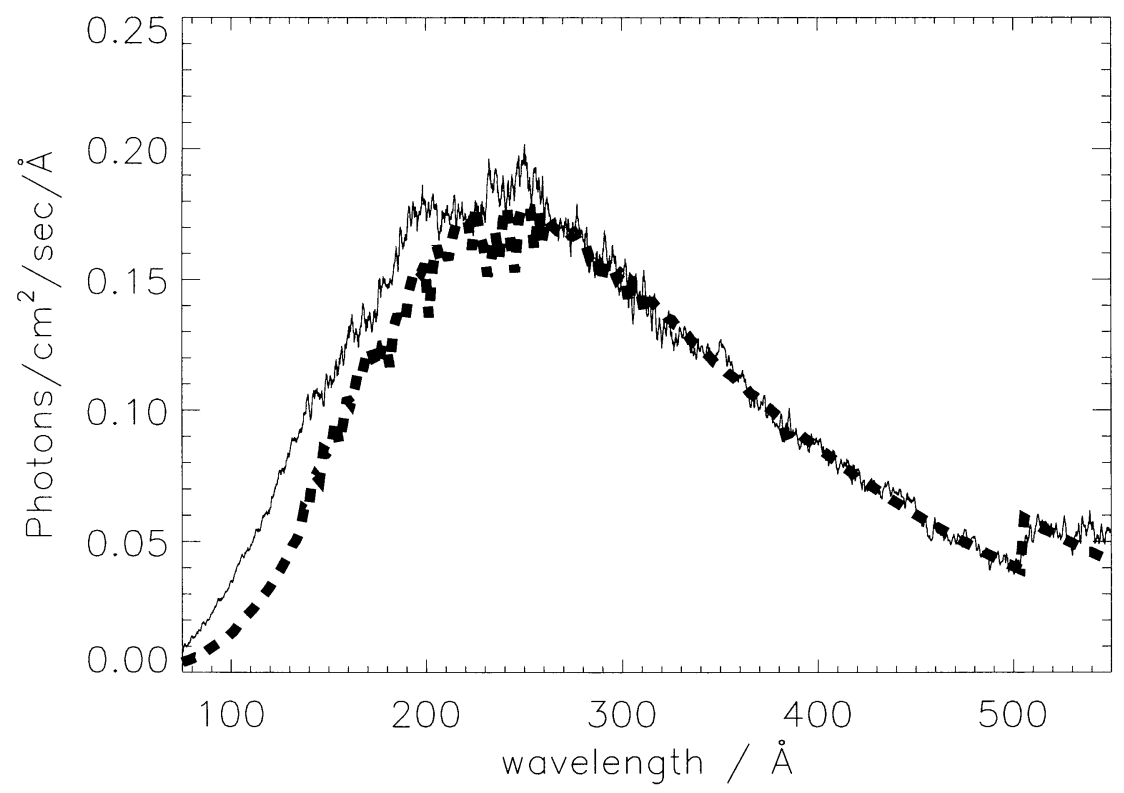

Figure 4. The EUVE spectrum of HZ $43 \mathrm{~A}$ (solid) compared with a stratified NLTE model atmosphere (dashed) of $T_{\text {eff }}=50000 \mathrm{~K}, \log g=$ 8.3 .

\section{Results}

In the recent years, we have presented our new chemically stratified model atmospheres as described above. We applied these model to hydrogen rich (DA) and hydrogen poor (DO) white dwarfs.

The application to 26 EUVE spectra of hot DA white dwarfs has revealed an overall good agreement (Dreizler \& Wolff 1999, Schuh et al. 2002), demonstrating the potential of these new models. Three representative examples are shown in Figs. 2 - 4. The agreements is good, however, some discrepancies are visible for the two cooler DAs. Whether these slight discrepancies can be traced back to the detailing of the models is currently investigated, but we want to stress the point again that the chemical composition is no longer a free parameter in these fits. Our results (Schuh et al. 2002) show that the abundance especially of iron is the dominating parameter for the EUV flux distribution. Fig. 5 displays sections of the HST-STIS UV spectrum of RE $1032+535$ covering C, N, and Si lines compared with a stratified NLTE model atmosphere. The agreement is very nice, again without adjusting the abundances.

In upcoming papers, we will check the derived parameters through a reanalysis of the Balmer lines and of the metal lines observable in UV spectra. The analysis of optical spectra with our new stratified models should allow to check for systematic errors as compared to previous analyses with chemically 

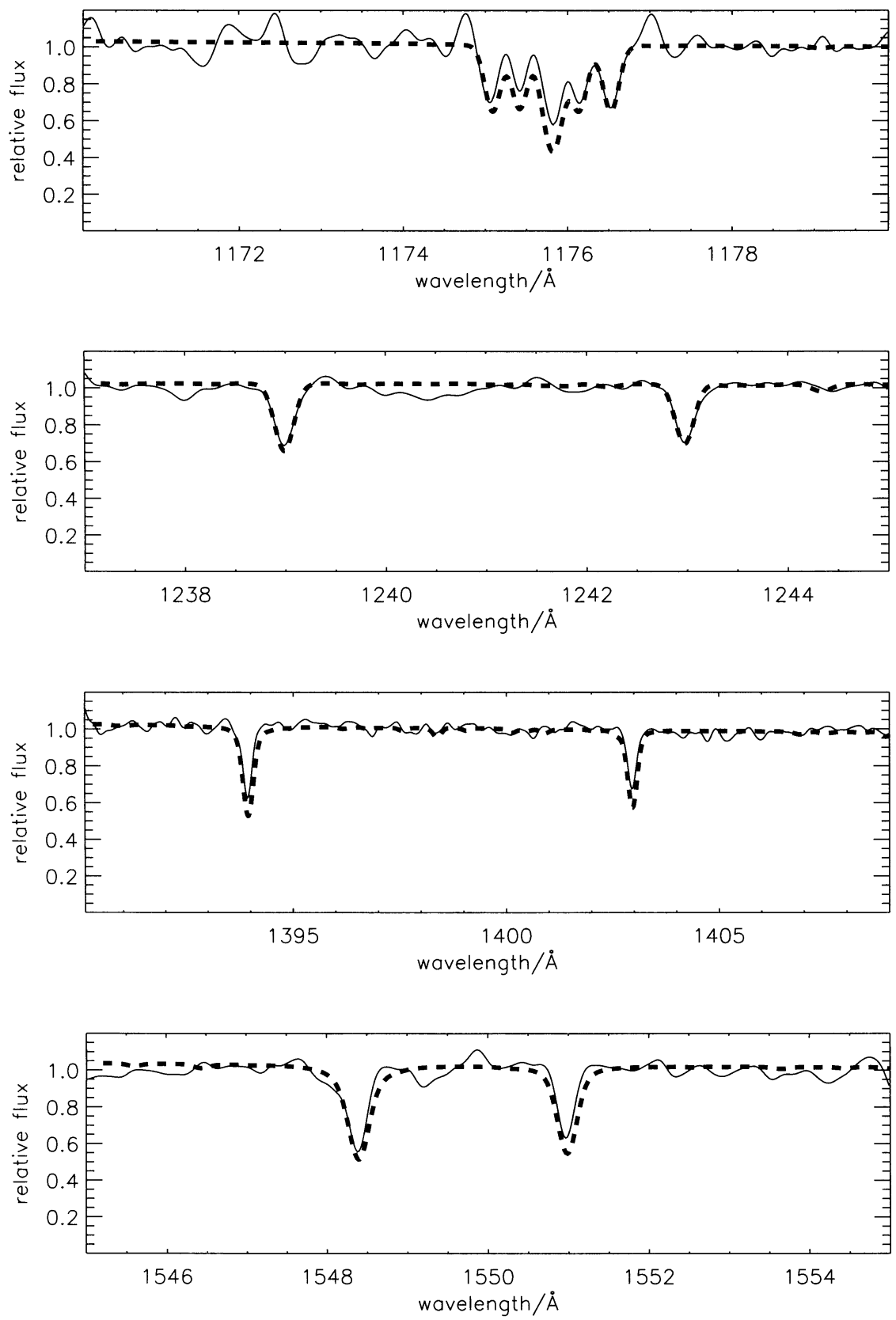

Figure 5. The HST-STIS UV spectrum of RE 1032+535 (solid) compared with a stratified NLTE model atmosphere (dashed) of $T_{\text {eff }}=$ $47000 \mathrm{~K}, \log g=7.8$. From top to bottom: CIII, NV, Si IV, CIV lines. 
homogeneous models. This will also be an important test for the consistency of atmospheric parameters derived from different parts of the spectrum, which has often posed a problem in the past.

The current models are only a first step, in the future we will be able to present an improved set of stratified model atmospheres. A "next generation" version of our stellar atmosphere code itself as well as a more efficient coupling between the computation of the model structure and the chemical stratification will allow us to include much more detailed model atoms and at the same time many more chemical elements. This is crucial for the accuracy of the calculation of the radiative acceleration. By evaluating the scale of the deviation in comparison to the current results it should then become clear how trustworthy the latter are.

The comparison for the DO white dwarfs is far less convincing (Dreizler 1999). The synthetic spectra of the stratified atmospheres were compared to UV (HST-GHRS) spectra of hot helium rich white dwarfs. These spectra were analyzed with chemically homogeneous as well as with stratified non-LTE model atmospheres. In the simple picture, where the chemical abundance of hot white dwarfs is determined through the interaction between gravitational settling and radiative levitation, the stratified models should result in a better fit. However, the stratified models reveal significant discrepancies whereas homogeneous models can nicely reproduce the observations. There are numerous possibilities to explain the failure of the stratified models in the current stage. The two most urgent ones, the treatment of the model atoms of the light metals and the inclusion of mass loss will be tackled in the future.

Acknowledgments. The authors would like to thank Klaus Werner (Tübingen) for useful comments and discussions. All model atmospheres have been calculated on CRAY machines of the Rechenzentrum der Universität Kiel. This work is supported by the Deutsche Forschungsgemeinschaft under grant DR 281/13-1 and 281/13-2. S.D. would also like to thank the organizers of the workshop for a financial support.

\section{Discussion}

WADE: We have recently begun calculating self-consistent chemically stratified model atmospheres for main sequence $A+B$ - type stars. We find that the assumptions of unlimited abundance source and the equilibrium condition $\log g_{\text {eff }}=0$ yield abundances which are systematically too large. Do you find similar problems?

DREIZLER: Yes, predicted abundances can be too high, especially in hot white dwarfs $\left(T_{\text {eff }} \geq 70000 \mathrm{~K}\right)$ where Fe comes out a factor of 10 too high. On the other hand it is not systematical in the sense that all abundances are predicted too high. 


\section{References}

Barstow, M. A., Fleming, T. A., \& Diamond, C. J., et al. 1993, MNRAS, 264, 16

Barstow, M. A., Dobbie, P. D., Holberg, J. B., Hubeny, I., \& Lanz, T. 1997, MNRAS, 286, 58

Barstow, M. A., Bannister, N. P., Holberg, J. B., Hubeny, I., Bruhweiler, F. C., \& Napiwotzki, R. 2001, MNRAS, 325, 1149

Bradley, P., \& Winget, D. 1994, ApJ, 430, 850

Bruhweiler F., \& Kondo Y. 1981, ApJ, 248, L123

Chayer P., Fontaine G., \& Wesemael F. 1989, in White Dwarfs, ed. G. Wegner, Springer, Berlin, p. 253

Chayer P., Fontaine G., \& Wesemael F. 1991, in White Dwarfs, eds. G. Vauclair, E. Sion, Kluwer, Dordrecht, p. 249

Chayer P., LeBlanc F., Fontaine G., et al. 1994, ApJ, 436, L161

Chayer P., Fontaine G., \& Wesemael F. 1995a, ApJS, 99, 189

Chayer P., Vennes S., Pradhan A.K., et al. 1995b, ApJ, 454, 429

Chayer, P., Kruk, J. W., Ake, T. B., Dupree, A. K., Malina, R. F., Siegmund, O. H. W., Sonneborn, G., \& Ohl, R. G. 2000, ApJ, 538, L91

Dreizler, S. 1999, A\&A, 352, 632

Dreizler, S. 2002, in Stellar Atmosphere Modeling, ASP Conference Series, ed.

I. Hubeny, D. Mihalas, \& K. Werner, in press

Dreizler, S., \& Wolff, B. 1999, A\&A, 348, 189

Greenstein, J. S., Truran, J. W., \& Cameron, A. G. W. 1967, Nature, 213, 871

Heber, U., Napiwotzki, R., \& Reid, I. N. 1997, A\&A, 323, 819

Holberg, J. B., Barstow, M. A., Bruhweiler, F. C., Hubeny, I., \& Green, E. M. 1999, ApJ, 517, 850

Hui-Bon-Hoa, A., LeBlanc, F., Hauschildt, P. H., \& Baron, E. 2002, A\&A, 381, 197

Jordan, S., Koester, D., Wulf-Mathies, C., \& Brunner, H. 1987, A\&A, 185, 253

Jordan, S., Wolff, B., Koester, D., \& Napiwotzki, R. 1994, A\&A, 290, 834

Kahn, S. M., Wesemael, F., Liebert, J., Raymond, J. C., Steiner, J. E., \& Shipman, H. L. 1984, ApJ, 278, 255

Kawaler, S. D., \& Bradley, P. A. 1994, ApJ, 427, 415

Koester, D., Dreizler, S., Weidemann, V., \& Allard, N. F. 1998, A\&A, 338, 612

Lampton, M., Margon, B., Paresce, F., Stern, R., \& Bowyer, S. 1976, ApJL, 203, L71

Lanz, T., Barstow, M. A., Hubeny, I., \& Holberg, J. B. 1996, ApJ, 473, 1089

Margon, B., Malina, R., Bowyer, S., Cruddace, R., \& Lampton, M. 1976, ApJ, 203, L25

Mewe, R., Heise, J., Gronenschild, E. H. B. M., Brinkman, A. C., Schrijver, J., \& den Boggende, A. J. F. 1975, ApJ, 202, L67

Michaud, G. 1970, ApJ, 160, 641 
Michaud, G., Vauclair G., \& Vauclair S. 1983, ApJ, 267256

Morvan E., Vauclair G., \& Vauclair S. 1986, A\&A, 163, 145

Paerels, F. B. S. \& Heise, J. 1989, ApJ, 339, 1000

Petre, R., Shipman, H. L., \& Canizares, C. R. 1986, ApJ, 304, 356

Schatzman, E. 1949, Publ. Köbenhavns Obs. No. 149

Schatzman, E. 1958, White Dwarfs, North Holland Publ. Comp., Amsterdam

Schuh, S. L., Dreizler, S., \& Wolff, B. 2002, A\&A, 382, 164

Shipman, H. L. 1976, ApJ, 206, L67

Sion E. M., Bohlin R. C., Tweedy R. W., \& Vauclair G. P. 1992, ApJ, 391, L29

Unglaub K., \& Bues I. 1998, A\&A 338, 75

Unglaub K., \& Bues I. 2000, A\&A 359, 1042

Unglaub K., \& Bues I. 2001, A\&A 374, 570

Vauclair G. 1987, in IAU Colloquium No. 95, The Second Conference on Faint Blue Stars, ed. A. G. D. Philip , D. S. Hayes, \& J. W. Liebert, L. Davis Press, Schenectady, p. 341

Vauclair G. 1989, in White Dwarfs, ed. G. Wegner, Springer, Berlin, p. 176

Vauclair G., Vauclair S., \& Greenstein J. L. 1979, A\&A 80, 79

Vennes, S., \& Lanz, T. 2001, ApJ, 553, 399

Vennes, S., Chayer, P., Fontaine, G., \& Wesemael, F. 1989, ApJL, 336, L25

Vennes S., Chayer P., Thorstensen J.R., Bowyer S., \& Shipman H.L. 1992, ApJ 392, L27

Vennes S., Chayer P., Hurwitz M., \& Bowyer S. 1996, ApJ 468, 898

Vidal-Madjar, A., Allard, N. F., Koester, D., Lemoine, M., Ferlet, R., Bertin, P., Lallement, R., \& Vauclair, G. 1994, A\&A, 287, 175

Werner, K., \& Dreizler, S. 1994, A\&A, 286, L31

Werner, K., \& Dreizler, S. 1999, in Computational Astrophysics, eds. H. Riffert \& K. Werner, Journal of Computational and Applied Mathematics, 109, 65

Wolff, B., Jordan, S., \& Koester, D. 1996, A\&A, 307, 149

Wolff, B., Koester, D., Dreizler, S., \& Haas, S. 1998, A\&A, 329, 1045 